\title{
ARTICLE
}

\section{AMPA receptor and metabotropic glutamate receptor 1 adaptations in the nucleus accumbens core during incubation of methamphetamine craving}

\author{
Conor H. Murray ${ }^{1}$, Jessica A. Loweth ${ }^{1,4}$, Mike Milovanovic ${ }^{1}$, Michael T. Stefanik ${ }^{1,5}$, Aaron J. Caccamise ${ }^{1,6}$, Hubert Dolubizno ${ }^{1,7}$, \\ Jonathan R. Funke ${ }^{1,2}$, M. Foster Olive ${ }^{3}$ and Marina E. Wolf ${ }^{1,2}$
}

Cue-induced drug craving progressively intensifies after withdrawal from self-administration of cocaine, methamphetamine, and other drugs of abuse, a phenomenon termed incubation of craving. For cocaine and methamphetamine, expression of incubated craving ultimately depends on strengthening of nucleus accumbens (NAc) synapses through an accumulation of high conductance $\mathrm{Ca}^{2+}$-permeable AMPA receptors (CP-AMPARs) that is detectable with electrophysiological approaches. This study sought to further characterize glutamate receptor adaptations in NAc core during methamphetamine incubation. Previous biochemical studies revealed that the CP-AMPARs accumulating after cocaine incubation are mainly homomeric GluA1 receptors and that their accumulation is reflected by increased cell surface GluA1. Here, for methamphetamine, we observed no significant change in surface or total GluA1 (GluA2 and GluA3 were also unchanged). Nonetheless, GluA1 translation was elevated after incubation of methamphetamine craving, as recently found for cocaine. Additionally, for cocaine, we previously observed a withdrawaldependent decrease in mGlu1 surface expression that precedes and enables CP-AMPAR accumulation and incubation of craving, reflecting weakening of mGlu1-dependent mechanisms that normally limit synaptic CP-AMPAR levels in the NAc core. Here, we observed no change in surface or total mGlu1 protein or its coupling to Homer scaffolding proteins after methamphetamine withdrawal, nor did elevation of mGlu1 tone through repeated injections of an mGlu1-positive allosteric modulator delay incubation of craving. These findings suggest a common role for increased GluA1 translation, but not decreased mGlu1 function, in the incubation of methamphetamine and cocaine craving. We speculate that increased GluA1 translation near synapses may drive formation and synaptic insertion of homomeric GluA1 receptors in the absence of detectable changes in GluA1 protein levels.

Neuropsychopharmacology (2019) 44:1534-1541; https://doi.org/10.1038/s41386-019-0425-5

\section{INTRODUCTION}

Persistent vulnerability to relapse is a major challenge in treating methamphetamine abuse. The majority of individuals discharged from methamphetamine treatment relapse within 1 year [1], yet there are currently no FDA-approved drugs to help recovering stimulant users stay abstinent. It is well established that methamphetamine abuse induces alterations in brain circuitry that are critical to relapse vulnerability, in part by modulating responses to drug-associated cues. In a rodent model termed the incubation model, relapse vulnerability is exemplified by a progressive increase or 'incubation' of cue-induced craving after withdrawal from self-administration of methamphetamine, as well as cocaine, heroin, nicotine, and ethanol $[2,3]$. For methamphetamine, incubation of craving is observed in rats during forced abstinence [4-6] or self-imposed abstinence [7], and in abstinent humans [8].
How do mechanisms underlying the incubation of methamphetamine craving compare to cocaine? One important point of convergence between the two drugs pertains to glutamate receptor plasticity in the nucleus accumbens (NAc) necessary for the expression of incubated craving. In drug-naïve or salinetreated animals, GluA2-containing $\mathrm{Ca}^{2+}$-impermeable a-amino-3hydroxy-5-methyl-4-isoxazole propionic acid receptors (AMPARs) are primarily responsible for excitatory transmission onto medium spiny neurons (MSNs), the major cell type and output neurons of the NAc $[9,10]$. However, following $\sim$ month of withdrawal from extended-access cocaine self-administration (i.e., after incubation of cocaine craving is near maximal), levels of high conductance $\mathrm{Ca}^{2+}$-permeable AMPA receptors (CP-AMPARs) are increased in NAc core and shell synapses; thereafter, their activation is required for expression of incubation [10, 11]. In NAc core, we showed that CP-AMPAR accumulation after cocaine is attributable to

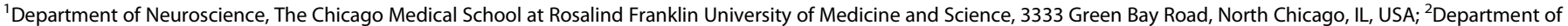
Behavioral Neuroscience, Oregon Health \& Science University, Portland, OR, USA and ${ }^{3}$ Department of Psychology, Arizona State University, Tempe, AZ, USA Correspondence: Marina E. Wolf (wolfmar@ohsu.edu)

${ }^{4}$ Present address: Department of Cell Biology and Neuroscience, Rowan University School of Osteopathic Medicine, Stratford, NJ, USA

${ }^{5}$ Present address: Department of Psychology and Neuroscience, North Central College, Naperville, IL, USA

${ }^{6}$ Present address: Department of Biomedical Sciences, Marquette University, Milwaukee, WI, USA

${ }^{7}$ Present address: Lake Erie College of Osteopathic Medicine, Erie, PA, USA

Received: 20 December 2018 Revised: 30 April 2019 Accepted: 20 May 2019

Published online: 30 May 2019 
increased homomeric GluA1 receptors, associated with an increase in cell surface GluA1 $[10,12]$ in the absence of significant changes in GluA2 [10]. The increase in surface GluA1 may be due to increased GluA1 translation [13].

In addition to CP-AMPAR accumulation after incubation of cocaine craving, a robust mGlu1-dependent synaptic depression mediated by the internalization of CP-AMPARs is detected $[14,15]$ and can be targeted to reduce cocaine craving [16-18]. The ability of mGlu1 activation to remove CP-AMPARs suggests that endogenous glutamate tone on mGlu1 may contribute to low CPAMPAR expression under control conditions, and that mGlu1 levels may be inversely related to CP-AMPAR levels. Indeed, we found that decreased mGlu1 surface expression in the NAc core precedes and enables CP-AMPAR accumulation during incubation of cocaine craving [17]. Furthermore, in a study of incubation of craving produced by a single cocaine self-administration session, a reduction in mGlu1 mRNA was found in NAc shell but not core, and an enhancement of incubated cocaine seeking was observed after systemic mGlu1 blockade [19].

In the NAc core of rats that undergo incubation of methamphetamine craving, electrophysiological studies have found that CP-AMPARs also accumulate, albeit more rapidly than following cocaine [5]. Once present, they are required for expression of incubation; furthermore, they are removed by acute pharmacological mGlu1 stimulation, leading to reduced craving [5]. In this study, we tested the hypothesis that CP-AMPAR accumulation during the incubation of methamphetamine craving involves increased translation and surface expression of GluA1 in the NAc core, similar to cocaine, and sought to determine whether CP-AMPAR accumulation after methamphetamine withdrawal is triggered by decreased mGlu1 expression. It is important to determine if common adaptations underlie cocaine and methamphetamine incubation because this could lead to common therapeutic approaches to maintain abstinence in individuals suffering from cocaine and methamphetamine addiction.

\section{MATERIALS AND METHODS}

Subjects and surgery

All procedures were approved by the Rosalind Franklin University of Medicine and Science and Oregon Health \& Science University IACUCs in accordance with the US Public Health Service Guide for Care and Use of Laboratory Animals. Male Sprague-Dawley rats (Envigo, Indianapolis, IN or South Kent, WA) weighing 275-300 g were housed 3/cage under a reverse 12-hour light/dark cycle with food and water available ad libitum. One week after arrival, rats were implanted with a jugular catheter under ketamine/xylazine anesthesia and thereafter were single-housed. Catheters were flushed daily with Cefazolin $(100 \mathrm{mg} / \mathrm{ml}$ in sterile $0.9 \%$ saline; 0.15 $\mathrm{ml}$ ) for the week prior to self-administration to prevent infection and maintain catheter patency.

Drug self-administration and cue-induced seeking tests Methamphetamine (from NIDA) was dissolved in $0.9 \%$ saline. Rats self-administered methamphetamine $(0.1 \mathrm{mg} / \mathrm{kg} ; 0.065 \mathrm{ml} /$ infusion $)$ or saline $(0.065 \mathrm{ml} /$ infusion) during 10 sessions (6 hours/day) conducted over 11-12 days with 1 or 2 days off in a chamber equipped with two nose-poke holes. Active hole nose-pokes resulted in an intravenous infusion paired with a 20-second light cue (white light illuminating the active hole) on a fixed-ratio 1 schedule. Each infusion was followed by a 20 -second time-out period. Nose poking in the inactive hole had no consequences. After 10 days of training, rats underwent 1-48 days of withdrawal in home cages and were handled weekly. This regimen produces incubation of cue-induced craving that is maximal after $\sim 1$ week of withdrawal [5], consistent with other findings [6]. One cohort of rats underwent cue-induced seeking tests, during which responding in the previously active hole delivered the light cue but no drug, in early and late withdrawal. Responding under these conditions is our operational measure of cue-induced methamphetamine craving.

\section{SYN119 Injections}

The selective mGlu1 positive allosteric modulator (PAM) SYN119 [(9H-Xanthene-9-carboxylic acid (4-trifluoromethyl-oxazol-2-ylamide)] was synthesized by EAG Labs (Tempe, AZ). SYN119 was dissolved in vehicle consisting of 10\% Tween-80 (vol/vol, \#P4780, Sigma-Aldrich, St. Louis, MO) in 0.9\% saline. Either SYN119 (10 mg/ $\mathrm{kg}$ ) or vehicle was injected intraperitoneally (i.p.) on withdrawal days (WD) 1,3,5, and 7 from methamphetamine self-administration, with no injections given on intervening days. Cue-induced seeking tests were conducted on WD1 (prior to the first SYN or vehicle injection) and on WD9.

\section{Biotinylation and co-immunoprecipitation}

Rats were decapitated and bilateral NAc core was dissected using a $1.5-\mathrm{mm}$ biopsy punch from two $1 \mathrm{~mm}$ slices prepared with a brain matrix. Tissue was biotinylated, as previously described [17], for analysis of surface GluA1, GluA2, GluA3, and mGlu1. Homogenates from the same rats were used for coimmunoprecipitation (co-IP) experiments, in which mGlu1 was immunoprecipitated and its associations with Homer $1 \mathrm{~b} / \mathrm{c}$ and Homer2 assessed with immunoblotting (see Supplementary Methods). Samples were run on 4-12\% Bis-Tris gels (Bio-Rad) and transferred to PVDF membranes as previously described $[10,12,17]$. Biotinylated tissue was immunoblotted with antibodies to GluA1 (1:500, sc-55509, Santa Cruz), GluA2 (1:300, clone L21/32, RRID: AB_10674575, UC Davis/NIH NeuroMab Facility, Davis, CA), GluA3 (1:300, 3437, Cell Signaling Technology, Danvers, MA), mGlu1 (1:10,000, 610964, BD Biosciences), Homer1b/c (1:200, sc-55463, Santa Cruz Biotechnology, Dallas, TX), or Homer2 (1:500, H00009455-B01P, Abnova, Taipei, Taiwan). The mGlu1 dimer band was analyzed because it represents the functional pool of these receptors [20]. For immunoblotting after immunoprecipitation, the same antibodies to Homer1b/c (1:200) and Homer2 (1:500) were used. Secondary antibodies to rabbit or mouse IgG light chain were used (211-032-171 or 115-035-174, Jackson ImmunoResearch Laboratories, West Grove, PA) in co-IP studies to prevent interference with the Homer band ( $\sim 5 \mathrm{kDa})$ by the heavy chain $(\sim 50 \mathrm{kDa})$. Proteins were visualized by chemiluminescence (ECL; GE Healthcare, Piscataway, NJ) and analyzed with TotalLab (Life Sciences Analysis Essentials) as described previously $[10,12,17]$. Data were excluded only if imperfections in the gel/blot interfered with analysis.

\section{Puromycin labeling}

On WD30 after methamphetamine or saline self-administration, NAc tissue (mainly core; see diagram in [21]) was dissected and newly translated proteins were labeled with $1 \mu \mathrm{M}$ puromycin (Sigma-Aldrich) for $1 \mathrm{~h}$, as detailed in Supplementary Methods and described previously [13]. Samples were immunoprecipitated with puromycin antibody ( $3 \mu \mathrm{g}, 3 \mathrm{RH} 11$, Kerafast, Boston, MA) to isolate newly translated proteins, and the immunoprecipitated fraction was immunoblotted with antibodies to GluA1 and GluA2 to measure newly translated pools of these subunits. This approach has been previously validated. First, side-by-side pulldowns with control IgG and puromycin antibody, followed by immunoblotting, verified that puromycin-labeled AMPAR subunit bands were not detected after IgG pulldown [13]. Second, preadsorption of GluA1 or GluA2 antibody with the peptide used to make the antibody, prior to immunoblotting, further verified the identity of the newly translated GluA1 and GluA2 bands (Stefanik, Milovanovic and Wolf, unpublished findings).

Statistical analyses

Cue-induced seeking on different WDs was compared using paired $t$-tests (two-tailed) except for the SYN119 study, which 
was analyzed with a mixed design module in Prism 8 (GraphPad Software Inc., La Jolla, San Jose, CA) with treatment (vehicle/ SYN119) as a between-group factor and withdrawal time (WD1/ WD9) as a within-group factor. For biochemical studies, unpaired two-tailed $t$-tests were performed. Data collection/analysis were not performed blind to experimental group. No statistical methods were used to predetermine sample sizes, but they are similar to those we used previously $[5,10,17]$.

\section{RESULTS}

Incubation of methamphetamine craving is not associated with changes in AMPAR subunit expression

To determine if the elevated CP-AMPAR transmission observed in whole-cell patch-clamp recordings of MSN after incubation of methamphetamine craving [5] is accompanied by changes in AMPAR subunit surface expression, we used biotinylation to measure cell surface and total protein levels of GluA1, GluA2, and GluA3 after withdrawal from methamphetamine or saline self-administration. Based on the time course of CP-AMPAR accumulation [5], biochemical analyses were conducted on WD3 and WD21 - withdrawal times at which CP-AMPAR accumulation is just beginning and maximally expressed, respectively. WD48 was also examined because this is a time-point at which craving is maximal and CP-AMPARs are stably elevated during incubation of cocaine craving [22]. Surprisingly, despite clear electrophysiological evidence for increased synaptic CP-AMPAR levels during incubation of methamphetamine craving [5], and robust increases in surface GluA1 accompanying CP-AMPAR accumulation during incubation of cocaine craving [10], we found no significant differences in total or cell surface AMPAR subunit expression at any withdrawal time between methamphetamine and saline groups (Fig. 1).

The rats depicted in Fig. 1 did not undergo cue-induced seeking tests to verify incubation of craving, although their training data
(Fig. S1) were in line with data from animals that exhibited incubation of craving after the identical regimen [5]. Nevertheless, to confirm our biochemical findings, we trained an additional cohort of saline and methamphetamine rats (Fig. 2a). Cue-induced seeking tests on WD1 and WD21 demonstrated incubation of craving $\left(t_{12}=3.20, p=0.008\right.$; Fig. $\left.2 b\right)$. In agreement with results in the test-naïve group (Fig. 1), surface GluA1 in NAc core tissue harvested and biotinylated on WD24 did not differ between saline and methamphetamine groups (Fig. 2c). It is possible that GluA1 subunits could be shifted towards formation of homomeric GluA1 receptors in the absence of detectable changes in GluA1 total protein or surface expression, particularly if the change is confined to synapses. In our prior cocaine studies, we used immunodepletion approaches as an alternate way to assess homomeric GluA1 receptors [10]. Unfortunately, due to loss of efficacy of a necessary antibody, our results for methamphetamine were inconclusive (Fig. S2).

\section{GluA1 and GluA2 translation after incubation of} methamphetamine craving

Accumulation of CP-AMPARs in the NAc after incubation of cocaine craving is accompanied by increased translation of GluA1 but not GluA2 [13]. Here, we measured GluA1 and GluA2 translation as we did for cocaine, utilizing the antibiotic puromycin, which resembles tyrosyl-transfer RNA and incorporates into newly translated proteins [23-25]. Although puromycin incorporation interrupts elongation, low concentrations of puromycin $(1 \mu \mathrm{M})$ sufficiently label proteins without substantially altering overall protein synthesis [24].

We prepared puromycin-labeled NAc tissue (from rats depicted in Fig. 1) and immunoprecipitated with puromycin antibody to isolate all newly translated (puromycin-containing) proteins on WD30, when CP-AMPARs are stably expressed in the NAc [5]. Immunoblotting for GluA1 and GluA2 proteins in the puromycinimmunoprecipitated fraction revealed a significant increase in

A

GluA1 Surface

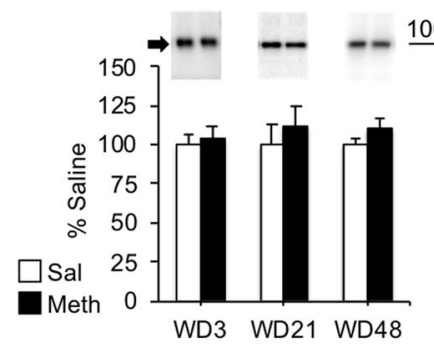

B

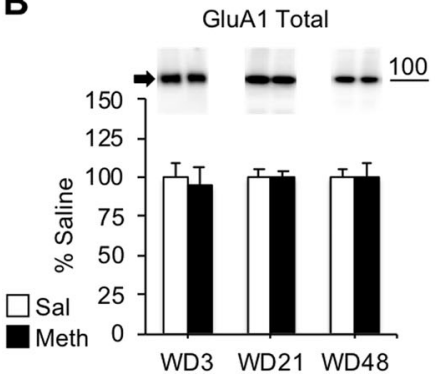

GluA2 Surface

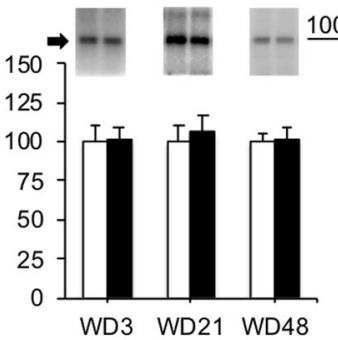

GluA2 Total

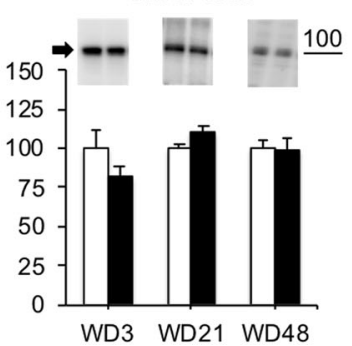

GluA3 Surface

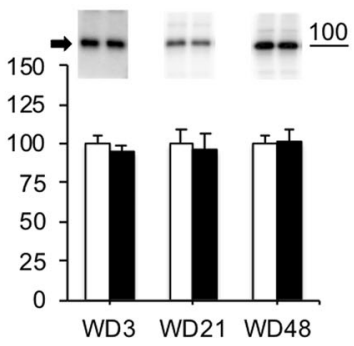

GluA3 Total

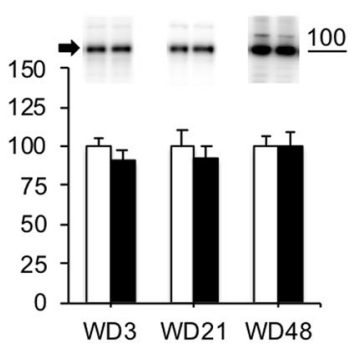

Fig. 1 Withdrawal from extended-access methamphetamine (Meth) self-administration is not associated with changes in AMPAR subunit expression in the NAc core. $\mathbf{a}$ Cell surface and $\mathbf{b}$ total protein levels of AMPAR subunits were measured in NAc core at three time-points after discontinuing self-administration of saline (Sal; control condition) or Meth: withdrawal day (WD) 3, WD21 and WD48. Data are expressed as \% control at each time-point ( $n=10-12$ rats/group; mean \pm SEM). Representative blots (cropped) are shown for a Sal rat (left) and a Meth rat (right) for each WD. Arrows indicate bands analyzed and lines indicate location of closest molecular weight marker (in kDa). We do not present full-length images because blots were cut to enable different molecular weight regions to be probed with different antibodies. The same applies to subsequent figures. Differences in optical density of bands for experiments conducted on different WDs reflect differences in exposure time 


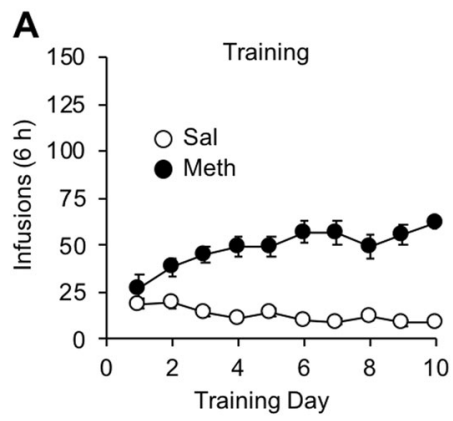

B
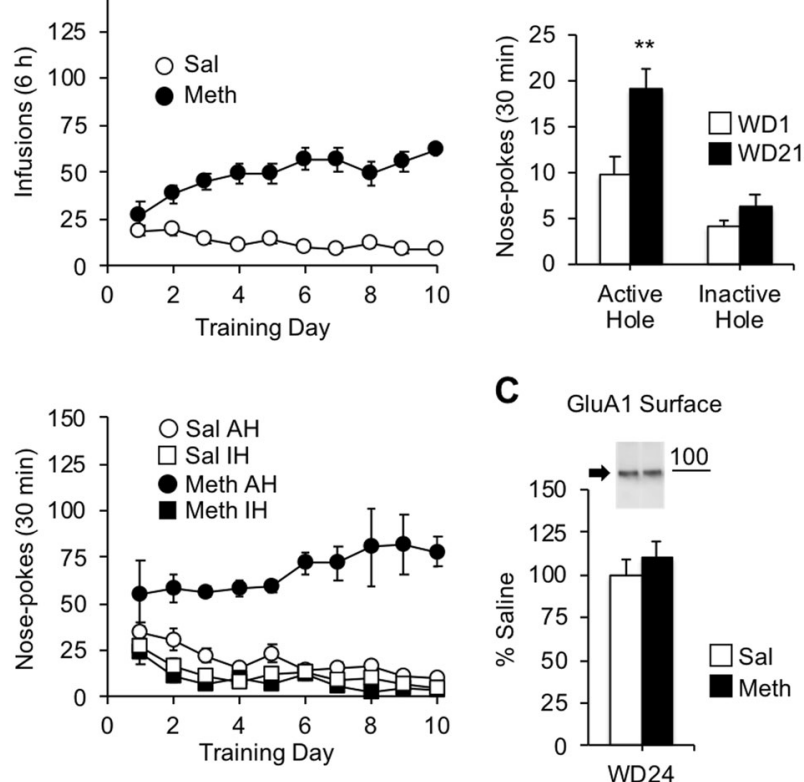

Fig. 2 Rats exhibiting incubation of cue-induced methamphetamine (Meth) seeking do not exhibit altered GluA1 surface expression in the NAc core. a Training. Rats self-administered saline or Meth for 6 hours/day for 10 days ( $n=7-8$ rats/group). Shown are the number of infusions (top) or number of active hole (AH) and inactive hole (IH) nose-pokes (bottom). b Seeking tests on withdrawal day (WD) 1 and WD21. During the $30 \mathrm{~min}$ test, $\mathrm{AH}$ nose-pokes led to presentation of the 20-second light cue previously paired with Meth infusion, but no Meth. Shown are AH and $\mathrm{IH}$ nose-pokes during the seeking test (paired $t$-test, ${ }^{* *} p<0.01$ WD1 vs WD21). c Cell surface levels of the AMPAR subunit GluA1 measured in NAc core on WD24, 3 days after the seeking test shown in $\mathbf{b}$. Data are expressed as percent of saline control. Representative blots (cropped) are shown for a Sal rat (left) and a Meth rat (right). Arrows indicate band analyzed and lines indicate location of closest molecular weight marker (in $\mathrm{kDa}$ ). All data are presented as mean \pm S.E.M.

newly translated GluA1 but not GluA2 in methamphetamine rats compared to saline controls $\left(t_{13}=2.22, p=0.045\right.$; Fig. 3 ), indicating a potential common mechanism for increased formation of homomeric GluA1 receptors after cocaine and methamphetamine withdrawal.

mGlu1 does not regulate CP-AMPAR accumulation during methamphetamine withdrawal

We found that decreased cell surface mGlu1 after cocaine withdrawal enables CP-AMPAR accumulation and incubation of craving. Thus, by restoring mGlu1 tone during a critical period of withdrawal (via every-other-day injections of the mGlu1 PAM SYN119), we prevented incubation and accumulation of CPAMPARs, although both resumed 2-3 days after the last PAM injection [17]. Here, we determined if mGlu1 plays a similar role during incubation of methamphetamine craving. We began by measuring cell surface and total mGlu1 on WD3, just prior to significant CP-AMPAR elevation, and on WD21 and WD48, when CP-AMPARs are stably elevated. To our surprise, saline and methamphetamine groups (same rats depicted in Fig. 1) did not differ in mGlu1 levels at any of these withdrawal times (Fig. 4). As reductions in Homer scaffolding proteins or their associations with mGlu1 could lead to reduced mGlu1 function (see Discussion), we assessed Homer1b/c and Homer2, two Homers that are expressed in the NAc and are altered by certain cocaine regimens [26, 27]. We measured total protein levels of these Homers (Fig. 5a) and

\section{Puromycin IP (WD30)}

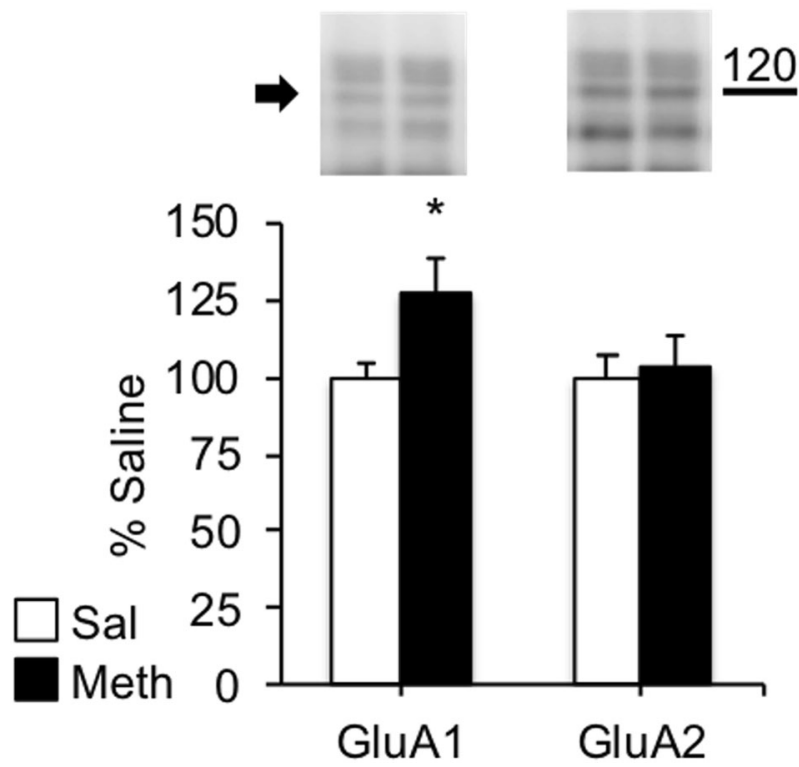

Fig. 3 GluA1 translation in the NAc is increased after 30 days of withdrawal from methamphetamine (Meth) self-administration compared to saline (Sal) controls while GluA2 translation is unchanged. NAc core tissue was incubated with puromycin to label newly translated proteins, immunoprecipitated using puromycin antibody, and then immunoblotted for GluA1 or GluA2. Data are expressed as \% control $(n=7-8$ rats/group; mean \pm SEM; unpaired $t$-test, ${ }^{*} p<0.05$ vs. saline). Representative blots (cropped) are shown for a Sal rat (left) and a Meth rat (right) for each AMPAR subunit. Arrows indicate bands analyzed and lines indicate location of closest molecular weight marker. Puromycin-labeled GluA1 and GluA2 subunits migrated slightly above their predicted molecular weight of $\sim 100 \mathrm{kDa}$, as shown previously [13]. Structural changes due to puromycin incorporation likely affect protein unfolding/SDS binding and thereby alter migration during SDS-PAGE
A

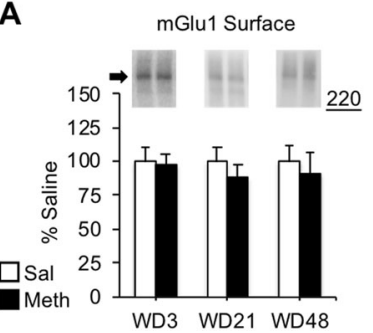

B

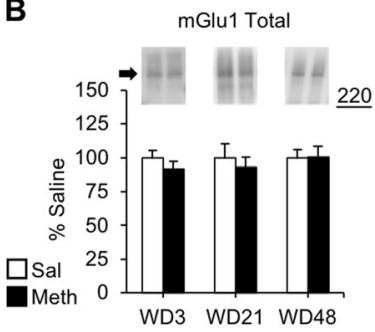

Fig. 4 Prolonged withdrawal from extended-access methamphetamine (Meth) self-administration does not affect expression of mGlu1 in the NAc core. a Surface and $\mathbf{b}$ total mGlu1 expression in NAc core were determined at three time-points during withdrawal from self-administration of saline (Sal; control condition) or Meth. Data are expressed as \% control ( $n=9-12$ rats/group; mean \pm SEM). No significant group differences were found. Representative blots (cropped) are shown for a Sal rat (left) and a Meth rat (right) for each withdrawal day (WD). Arrows indicate bands analyzed and lines indicate location of closest molecular weight marker. The mGlu1 dimer band was analyzed because it represents the functional pool of these receptors [20]. Please note that differences in optical density of bands for experiments conducted on different WDs reflect differences in exposure time

also assessed their association with mGlu1 by immunoprecipitating mGlu1 and measuring bound Homer levels (Fig. 5b). No differences between saline and methamphetamine groups were found at any withdrawal time. 
A

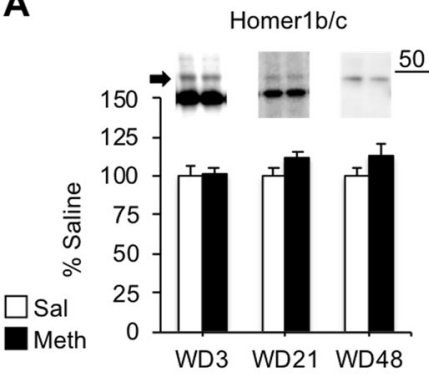

B

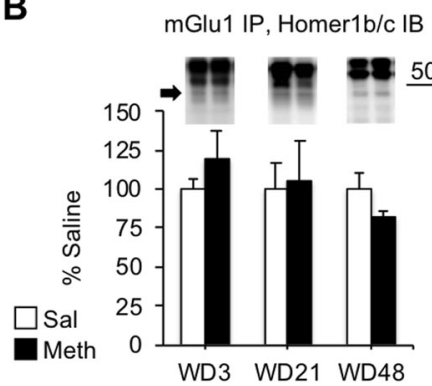

Homer2

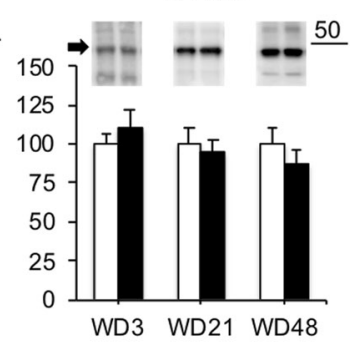

mGlu1 IP, Homer2 IB

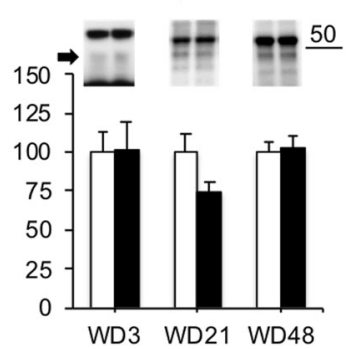

Fig. 5 Prolonged withdrawal from extended-access methamphetamine (Meth) self-administration does not alter total Homer levels or mGlu1-Homer associations compared to saline (Sal) controls. a Total Homer protein expression from NAc core homogenates, expressed as \% of control ( $n=12$ rats/group; mean \pm SEM). b Coimmunoprecipitation experiments assessing the physical associations between mGlu1 and Homer scaffolding proteins at three timepoints during withdrawal from self-administration of Sal or Meth ( $n=9-12$ rats/group, same rats as shown in panel a; mean \pm SEM). Representative blots (cropped) are shown for a Sal rat (left) and a Meth rat (right) for each withdrawal day (WD). Arrows indicate Homer bands $(\sim 45 \mathrm{kDa})$ and lines indicate location of closest molecular weight marker. Please note that differences in optical density of bands between experiments conducted on different WDs reflect differences in exposure time. IB, immunoblot; IP, immunoprecipitation.

Despite lack of evidence for reduced mGlu1 function after methamphetamine withdrawal, we wondered whether enhancing mGlu1 tone during the rising phase of incubation might nevertheless delay or suppress incubation. To test this, methamphetamine rats were injected with SYN119 $(10 \mathrm{mg} / \mathrm{kg}$, i.p.) every other day during the rising phase of incubation and CP-AMPAR accumulation (i.e., on WD1, WD3, WD5, and WD7). Cue-induced seeking tests were administered on WD1, to define baseline craving, and on WD9, to determine if incubation had occurred (Fig. 6). Analysis with a mixed design module revealed an effect of withdrawal time $\left(F_{1,14}=9.04, p=0.009\right)$ but no significant treatment effect $\left(F_{1,14}=0.27, p=0.609\right)$ or treatment $\times$ withdrawal day interaction $\left(F_{1,14}=0.07, p=0.799\right)$. Thus, in contrast to cocaine studies, repeated SYN119 treatment during withdrawal did not prevent the incubation of methamphetamine craving.

\section{DISCUSSION}

Cocaine and methamphetamine exhibit both similarities and differences. While both are monoamine reuptake inhibitors, methamphetamine also releases monoamines by reversing transporter function [28], methamphetamine is neurotoxic to dopamine and serotonin neurons [29], and methamphetamine clearance is substantially slower $[30,31]$. Recently, acute administration of cocaine and methamphetamine to rats was shown to produce very different effects on the blood-oxygenation-level dependent (BOLD) signal throughout the reward circuit [32].

A

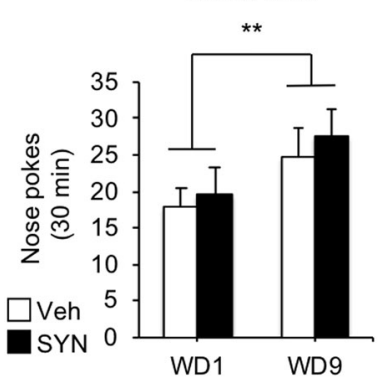

B

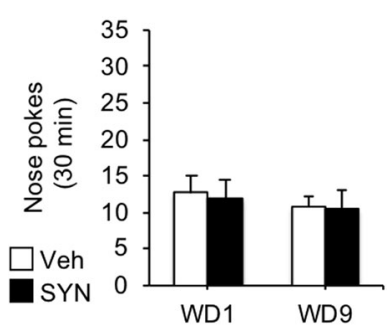

Fig. 6 Repeated systemic injections of the mGlu1 positive allosteric modulator SYN119 (SYN) during early withdrawal do not affect incubation of cue-induced methamphetamine seeking. For each rat, seeking tests (30 $\mathrm{min}$ ) were conducted on withdrawal day (WD) 1 and WD9 after methamphetamine self-administration. SYN119 (10 $\mathrm{mg} / \mathrm{kg}$, i.p.) or vehicle (Veh) was injected every other day from WD1 (following the WD1 seeking test) through WD7. Shown are nosepokes in the previously active hole (a) and the inactive hole (b) during the seeking test. Nose-pokes in the previously active hole led to presentation of the 20 -second light cue previously paired with each methamphetamine infusion. Data are presented as mean \pm SEM ( $n=8$ rats/group; mixed design module, significant effect of withdrawal time: ${ }^{* *} p<0.01$ )

However, after discontinuing drug self-administration, incubation of cue-induced craving occurs that is of similar magnitude for cocaine and methamphetamine [2]. This, combined with the ability of other classes of drugs of abuse to also produce incubation [2], suggests a dominant role for withdrawaldependent plasticity as opposed to acute drug actions in eliciting incubation. The goal of this study was to determine if glutamate receptor-related adaptations in the NAc core during incubation of methamphetamine craving are similar to those previously characterized for cocaine. A limitation of our study is that we were unable to compare these changes in D1 and D2 receptorexpressing MSNs, but considerable evidence indicates that, in NAC core, both populations undergo similar CP-AMPAR plasticity during incubation of cocaine craving [11]. Our results reveal a shared increase in GluA1 translation in the NAc after incubation of cocaine and methamphetamine craving.

\section{AMPAR expression during incubation of methamphetamine craving}

We began by studying expression of AMPAR subunits GluA1-3 using NAc core tissue from saline and methamphetamine rats obtained at three time-points (WD3, WD21, and WD48). We focused on NAc core because it is critical in cue-induced cocaine seeking [33] and because blockade or removal of CP-AMPARs in the NAc core is sufficient to inhibit the expression of incubated craving for cocaine $[10,17]$ or methamphetamine [5].

Whole-cell patch-clamp recordings detect elevated CP-AMPAR levels in NAc core after incubation of both cocaine and methamphetamine craving $[5,10]$. For cocaine, this is accompanied by increased cell surface GluA1; other biochemical approaches (e.g., co-immunoprecipitation) further indicate that the CP-AMPARs detected with electrophysiology are mainly homomeric GluA1 receptors [10, 12]. Cell surface GluA3 is also modestly increased after cocaine self-administration, but in a withdrawal-independent manner (i.e., it was elevated on both WD1 and WD45 in cocaine vs saline rats) [10]. Surprisingly, the present study found no change in cell surface or total GluA1, GluA2, or GluA3 in methamphetamine rats. We attempted coimmunoprecipitation studies akin to those performed for cocaine [10], but results were inconclusive (Fig. S2). These results suggest that CP-AMPAR accumulation after methamphetamine craving must be associated with subtle biochemical changes, perhaps 
confined to synapses and therefore not detected with our methods. In theory, such a change could be revealed by analyzing a postsynaptic density (PSD) fraction. However, in our studies of NAc tissue from rats that had undergone incubation of cocaine craving, we found that, despite robust increases in GluA1 in biotinylated and LP1 fractions, this enrichment was not preserved in a Triton insoluble PSD fraction, indicating that the CP-AMPARs added during incubation, while synaptic, are not tightly anchored within the PSD [12].

A study conducted 8 days after discontinuing a similar methamphetamine regimen revealed no change in the AMPA/ NMDA ratio in NAc core MSN [34]. Insertion of high conductance CP-AMPARs would have been expected to increase this ratio; potential explanations include minor differences between regimens and the possibility of concurrently increased NMDAR transmission. It should be noted that, although we have focused on postsynaptic glutamate receptors, others have found changes in extracellular glutamate levels [35], mGlu2/3 expression [36], and membrane excitability [37] in the NAc after withdrawal from methamphetamine self-administration.

mGlu1 and incubation of methamphetamine craving

In the NAC of rats that have undergone incubation of cocaine craving, as well as at other synapses containing appreciable $\mathrm{CP}$ AMPAR levels, mGlu1 stimulation elicits a form of LTD that depends on CP-AMPAR internalization (see ref. [38] for review, and refs. $[14,15])$. Triggering this LTD via an acute injection of SYN119 (shortly before a seeking test) removes CP-AMPARs from NAc core synapses and thereby blocks the expression of incubated cueinduced cocaine [17] or methamphetamine craving [5]. mGlu1 also plays an important role in the rising phase of incubation of cocaine craving. Thus, decreased mGlu1 surface expression is detected on WD25 (just as CP-AMPAR levels begin to rise) and WD48 (when they are stably expressed), but not as early as WD14 (prior to any alteration in CP-AMPARs) [17]. These results suggest that reduced mGlu1 surface expression, resulting in reduced mGlu1-mediated CP-AMPAR internalization $[14,15]$ and perhaps increased translation of GluA1 [39], is permissive for CP-AMPAR accumulation during incubation of cocaine craving. Does the same apply to methamphetamine?

Given that CP-AMPAR accumulation and incubation occur more rapidly after methamphetamine withdrawal, with both approaching maximal levels by WD7-8 [5, 6], we hypothesized that a decrease in NAc core mGlu1 surface expression may occur during the first few days of methamphetamine withdrawal. However, no changes in mGlu1 surface or total protein expression were detected on WD3, or at later withdrawal time-points. Furthermore, potentiating mGlu1 transmission with SYN119 during the rising phase of incubation and CP-AMPAR accumulation did not prevent the incubation of methamphetamine craving. These results, taken together with previous findings, indicate that reduced mGlu1 function in early withdrawal enables incubation of cocaine craving but not methamphetamine craving.

Despite lack of any decrease in mGlu1 surface expression after methamphetamine, it remained possible that mGlu1 function was decreased in early withdrawal due to a reduction in Homer proteins or their association with mGlu1. Three Homer genes (Homer1, Homer2, and Homer3) encode transcriptional variants that include constitutively expressed long forms (Homer1b-d, Homer2ab, and Homer3) and truncated activity-induced forms (Homer1a, ania-3). Long Homers consist of a coiled-coil domain that enables dimerization and facilitates coupling between group I mGluRs and downstream effectors [27, 40]. Thus, a reduction in Homer proteins or their association with mGlu1 could lead to reduced mGlu1 function and subsequent CP-AMPAR accumulation during incubation. In the NAc, reductions in Homer protein levels have been found following cocaine exposure [27], including decreases in Homer1b/c and Homer2 following extended-access cocaine self-administration [26]. Here, we did not observe changes in total Homer $1 \mathrm{~b} / \mathrm{c}$ or Homer2 levels, or mGlu1-Homer associations, during incubation of methamphetamine craving. We note that mGlu1-Homer associations were also unaffected by incubation of cocaine craving [17].

\section{GluA1 translation}

Newly translated GluA1 was labeled by incubating NAc tissue with puromycin, which incorporates into nascent polypeptides at tyrosine codons [23-25], and measured by immunoblotting for GluA1 following immunoprecipitation with puromycin antibody. Recently, we used this approach to demonstrate increased translation of GluA1 (but not GluA2) in cocaine rats compared to saline controls on $>$ WD40, when CP-AMPAR levels are stably elevated, but not on WD1, prior to their elevation [13]. Similarly, at a withdrawal time when CP-AMPARs are stably increased after incubation of methamphetamine craving (WD30), levels of newly translated GluA1 but not GluA2 were elevated in methamphetamine rats compared to saline controls, suggesting that CP-AMPAR accumulation during incubation of both methamphetamine and cocaine craving may stem at least in part from increased GluA1 translation. This is consistent with prior results showing that maintenance of elevated CP-AMPAR levels in the $N A c$ of rats that have undergone incubation of cocaine craving requires ongoing protein translation [41] and that protein translation is dysregulated during incubation of cocaine craving $[13,42]$.

Other pathways implicated in methamphetamine incubation Although we have focused on NAc, incubation involves additional brain regions that exhibit both similarities and differences related to cocaine versus methamphetamine incubation. Notably, activation (measured with c-Fos) of the ventromedial prefrontal cortex (vmPFC) and central nucleus of the amygdala $(\mathrm{CeA})$ is necessary for expression of cocaine incubation, while the $\mathrm{CeA}$, but not the vmPFC, is necessary for expression of methamphetamine incubation [43]. Neurochemical studies also implicate the PFC in cocaine incubation [44]. Extending the CeA findings, a genome-wide transcriptional profiling study of $\mathrm{CeA}$ during incubation of methamphetamine craving found substantially more differentially expressed genes on WD35 than WD2; biological processes overrepresented in the upregulated genes on WD35 included postsynapse organization and histone lysine methylation [45]. In orbitofrontal cortex, which is not required for expression of methamphetamine incubation but is implicated in other aspects of addiction [43], there were fewer differentially expressed genes overall and more upregulated genes on WD2 than WD35, as well as an interesting overlap between upregulated genes on WD2 and downregulated genes on WD35 [45].

Studies on methamphetamine incubation have also revealed a role for dorsal striatum (DS). No changes in glutamate receptor mRNAs were evident when comparing DS homogenates from rats that underwent a cue-induced seeking test on WD2 versus WD35. However, separation of Fos-positive (cue-activated) and Fos-negative neurons using fluorescence-activated cell sorting revealed that the Fos-positive population (which included both D1 and D2 receptor-expressing neurons) expressed increased levels of mRNA coding for GluA1, GluA3, GluN2A, and mGlu1 (mRNA for GluA2 was decreased), as well as increases in mRNA for epigenetic enzymes [46]. Expression of incubation on WD35 required D1 receptor signaling (D2 antagonists were not tested) and histone deacetylase $5[46,47]$. These findings have been extended to show that glutamatergic projections from the anterior intralaminar nuclei of the thalamus interact with D1 receptor-mediated signaling in DS to promote methamphetamine craving [48]. It will be important to investigate this pathway for cocaine as some evidence suggests a role for DS in incubation of cocaine craving [11]. Given the commonality between 
methamphetamine and cocaine incubation in regard to $\mathrm{CP}$ AMPAR accumulation in NAc core, and perhaps a role for the DS, it is interesting to speculate that NAc core and DS may work together to enable expression of incubation, as previously shown for cue-maintained cocaine seeking [49].

\section{CONCLUSION}

These experiments compared glutamatergic plasticity in NAc core between the incubation of cocaine and methamphetamine craving. Although AMPAR subunit and mGlu1 expression were unaltered during methamphetamine incubation, and mGlu1 potentiation during withdrawal was insufficient to prevent methamphetamine incubation, a commonality was found in increased GluA1 translation, suggesting a potential explanation for accumulation of homomeric GluA1 CP-AMPARs during incubation of craving for both drugs. Our ability to detect CPAMPARs using electrophysiological methods [5], but not biochemical methods (present study), suggests subtler biochemical alterations underlying CP-AMPAR accumulation in methamphetamine incubation. Relevant to this, we note that the newly translated pool of GluA1 is a small fraction of total GluA1 in the NAc [13]. If new GluA1 translation preferentially supplies synaptic pools, it could influence synaptic transmission in the absence of biochemically detectable changes in GluA1 protein. Future efforts should focus on testing a causal relationship between increased GluA1 translation and increased synaptic CP-AMPARs, and on common mechanisms supporting elevated GluA1 translation in methamphetamine and cocaine incubation.

\section{FUNDING AND DISCLOSURE}

This work was supported by US Public Health Service grants DA009621 (M.E.W.), DA043172 and DA024355 (M.F.O.), F32 DA040414 (M.T.S), and Pathway to Independence Award K99 DA038110 (J.A.L.). The authors declare no competing interests.

\section{ACKNOWLEDGEMENTS}

We thank Dr. Katherine Roche for generously supplying the GluA2/3 antibody and Dr. Pamela Metten for advice on statistical analysis.

\section{ADDITIONAL INFORMATION}

Supplementary Information accompanies this paper at (https://doi.org/10.1038/ s41386-019-0425-5)

Publisher's note: Springer Nature remains neutral with regard to jurisdictional claims in published maps and institutional affiliations.

\section{REFERENCES}

1. Brecht $M-L$, Herbeck D. Time to relapse following treatment for methamphetamine use: a long-term perspective on patterns and predictors. Drug Alcohol Depend. 2014;139:18-25.

2. Pickens $C L$, Airavaara M, Theberge F, Fanous S, Hope BT, Shaham Y. Neurobiology of the incubation of drug craving. Trends Neurosci. 2011;34:411-20.

3. Lu L, Grimm JW, Hope BT, Shaham Y. Incubation of cocaine craving after withdrawal: a review of preclinical data. Neuropharmacology. 2004;47:214-26.

4. Shepard JD, Bossert JM, Liu SY, Shaham Y. The anxiogenic drug yohimbine reinstates methamphetamine seeking in a rat model of drug relapse. Biol Psychiatry. 2004;55:1082-9.

5. Scheyer AF, Loweth JA, Christian DT, Uejima J, Rabei R, Le T, et al. AMPA receptor plasticity in accumbens core contributes to incubation of methamphetamine craving. Biol Psychiatry. 2016;0:5-15.

6. Adhikary $S$, Caprioli $D$, Venniro $M$, Kallenberger $P$, Shaham $Y$, Bossert JM. Incubation of extinction responding and cue-induced reinstatement, but not context- or drug priming-induced reinstatement, after withdrawal from methamphetamine. Addict Biol. 2017;22:977-90.
7. Venniro M, Caprioli D, Shaham Y. Animal models of drug relapse and craving: From drug priming-induced reinstatement to incubation of craving after voluntary abstinence. Prog Brain Res. 2016;224:25-52. https://doi.org/10.1016/bs. pbr.2015.08.004.

8. Wang G, Shi J, Chen N, Xu L, Li J, Li P, et al. Effects of length of abstinence on decision-making and craving in methamphetamine abusers. PLoS ONE. 2013;8: e68791.

9. Kourrich S, Rothwell PE, Klug JR, Thomas MJ. Cocaine Experience controls bidirectional synaptic plasticity in the nucleus accumbens. J Neurosci. 2007;27:7921-8.

10. Conrad KL, Tseng KY, Uejima JL, Reimers JM, Heng LJ, Shaham Y, et al. Formation of accumbens GluR2-lacking AMPA receptors mediates incubation of cocaine craving. Nature. 2008;454:118-21.

11. Wolf ME. Synaptic mechanisms underlying persistent cocaine craving. Nat Rev Neurosci. 2016;17:351-65.

12. Ferrario $C R$, Loweth JA, Milovanovic M, Ford KA, Galiñanes GL, Heng L-J, et al. Alterations in AMPA receptor subunits and TARPs in the rat nucleus accumbens related to the formation of $\mathrm{Ca2}^{+}$- permeable AMPA receptors during the incubation of cocaine craving. Neuropharmacology. 2011;61:1141-51.

13. Stefanik MT, Milovanovic M, Werner CT, Spainhour JCG, Wolf ME. Withdrawal from cocaine self-administration alters the regulation of protein translation in the nucleus accumbens. Biol Psychiatry. 2018;84:223-32.

14. McCutcheon JE, Loweth JA, Ford KA, Marinelli M, Wolf ME, Tseng KY. Group I mGluR activation reverses cocaine-induced accumulation of calcium-permeable AMPA receptors in nucleus accumbens synapses via a protein kinase C-dependent mechanism. J Neurosci. 2011;31:14536-41.

15. Scheyer AF, Christian DT, Wolf $M$, Tseng KY. Emergence of endocytosisdependent mGlu1 LTD at nucleus accumbens synapses after withdrawal from cocaine self-administration. Front Synaptic Neurosci. 2018;10:36.

16. Lee BR, Ma $Y-Y$, Huang $Y H$, Wang $X$, Otaka $M$, Ishikawa $M$, et al. Maturation of silent synapses in amygdala-accumbens projection contributes to incubation of cocaine craving. Nat Neurosci. 2013;16:1644-51.

17. Loweth JA, Scheyer AF, Milovanovic M, LaCrosse AL, Flores-Barrera E, Werner CT, et al. Synaptic depression via mGluR1 positive allosteric modulation suppresses cue-induced cocaine craving. Nat Neurosci. 2014;17:73-80.

18. Ma YY, Lee BR, Wang X, Guo C, Liu L, Cui $R$, et al. Bidirectional modulation of incubation of cocaine craving by silent synapse-based remodeling of prefrontal cortex to accumbens projections. Neuron. 2014;83:1453-67.

19. Halbout B, Bernardi RE, Hansson AC, Spanagel R. Incubation of cocaine seeking following brief cocaine experience in mice is enhanced by mGluR1 blockade. J Neurosci. 2014;34:1781-90.

20. Jingami $H$, Nakanishi $S$, Morikawa K. Structure of the metabotropic glutamate receptor. Curr Opin Neurobiol. 2003;13:271-8.

21. McCutcheon JE, Wang $X$, Tseng KY, Wolf ME, Marinelli M. Calcium-permeable AMPA receptors are present in nucleus accumbens synapses after prolonged withdrawal from cocaine self-administration but not experimenter-administered cocaine. J Neurosci. 2011;31:5737-43.

22. Wolf ME, Tseng KY. Calcium-permeable AMPA receptors in the VTA and nucleus accumbens after cocaine exposure: when, how, and why? Front Mol Neurosci. 2012;5:72.

23. Schmidt EK, Clavarino G, Ceppi M, Pierre P. SUnSET, a nonradioactive method to monitor protein synthesis. Nat Methods. 2009;6:275-7.

24. Goodman CA, Hornberger TA. Measuring protein synthesis with SUnSET: a valid alternative to traditional techniques? Exerc Sport Sci Rev. 2013:41:107-15.

25. Starck SR, Green HM, Alberola-lla J, Roberts RW. A general approach to detect protein expression in vivo using fluorescent puromycin conjugates. Chem Biol. 2004;11:999-1008.

26. Ben-Shahar O, Obara I, Ary AW, Ma N, Mangiardi MA, Medina RL, et al. Extended daily access to cocaine results in distinct alterations in Homer $1 \mathrm{~b} / \mathrm{c}$ and NMDA receptor subunit expression within the medial prefrontal cortex. Synapse. 2009;63:598-609.

27. Szumlinski KK, Ary AW, Lominac KD. Homers regulate drug-induced neuroplasticity: implications for addiction. Biochem Pharm. 2008;75:112-33.

28. Sulzer D. How addictive drugs disrupt presynaptic dopamine neurotransmission. Neuron. 2011;69:628-49.

29. Halpin LE, Collins SA, Yamamoto BK. Neurotoxicity of methamphetamine and 3,4-methylenedioxymethamphetamine. Life Sci. 2014;97:37-44.

30. Rivière GJ, Gentry WB, Owens SM. Disposition of methamphetamine and its metabolite amphetamine in brain and other tissues in rats after intravenous administration. J Pharm Exp Ther. 2000;292:1042-7.

31. Tsibulsky VL, Norman AB. Satiety threshold: a quantitative model of maintained cocaine self-administration. Brain Res. 1999:839:85-93.

32. Taheri S, Xun Z, See RE, Joseph JE, Reichel CM. Cocaine and methamphetamine induce opposing changes in BOLD signal response in rats. Brain Res. 2016;1642: 497-504. 
AMPA receptor and metabotropic glutamate receptor 1 adaptations in the... $\mathrm{CH}$ Murray et al.

33. Milton AL, Everitt BJ. The persistence of maladaptive memory: addiction, drug memories and anti-relapse treatments. Neurosci Biobehav Rev. 2012;36:1119-39.

34. Mishra D, Pena-Bravo Jl, Leong K-C, Lavin A, Reichel CM. Methamphetamine self-administration modulates glutamate neurophysiology. Brain Struct Funct. 2017;222:2031-9.

35. Lominac KD, Sacramento AD, Szumlinski KK, Kippin TE. Distinct neurochemical adaptations within the nucleus accumbens produced by a history of selfadministered vs non-contingently administered intravenous methamphetamine. Neuropsychopharmacology. 2012;37:707-22.

36. Schwendt M, Reichel CM, See RE, Hashimoto K. Extinction-dependent alterations in corticostriatal mGluR2/3 and mGluR7 receptors following chronic methamphetamine self-administration in rats. 2012. https://doi.org/10.1371/journal.pone.0034299.

37. Graves SM, Clark MJ, Traynor JR, Hu X-T, Napier TC. Nucleus accumbens shell excitability is decreased by methamphetamine self-administration and increased by $5-\mathrm{HT} 2 \mathrm{C}$ receptor inverse agonism and agonism. Neuropharmacology. 2015;89:113-21.

38. Loweth JA, Tseng KY, Wolf ME. Using metabotropic glutamate receptors to modulate cocaine's synaptic and behavioral effects: mGluR1 finds a niche. Curr Opin Neurobiol. 2013;23:500-6.

39. Loweth JA, Reimers JM, Caccamise A, Stefanik MT, Woo K-Y, Chauhan NM, et al. mGlu1 tonically regulates levels of calcium-permeable AMPA receptors in cultured nucleus accumbens neurons through retinoic acid signaling and protein translation. Eur J Neurosci. 2018. https://doi.org/10.1111/ ejn.14151.

40. Xiao B, Tu JC, Petralia RS, Yuan JP, Doan A, Breder CD, et al. Homer regulates the association of group 1 metabotropic glutamate receptors with multivalent complexes of homer-related, synaptic proteins. Neuron. 1998;21:707-16.

41. Scheyer AF, Wolf ME, Tseng KY. A protein synthesis-dependent mechanism sustains calcium-permeable AMPA receptor transmission in nucleus accumbens synapses during withdrawal from cocaine self-administration. J Neurosci. 2014;34:3095-3100.

42. Werner CT, Stefanik MT, Milovanovic M, Caccamise A, Wolf ME. Protein translation in the nucleus accumbens is dysregulated during cocaine withdrawal and required for expression of incubation of cocaine craving. J Neurosci. 2018;38: 2683-97.

43. Li X, Zeric T, Kambhampati S, Bossert JM, Shaham Y. The central amygdala nucleus is critical for incubation of methamphetamine craving. Neuropsychopharmacology. 2015;40:1297-306.

44. Shin CB, Templeton TJ, Chiu AS, Kim J, Gable ES, Vieira PA, et al. Endogenous glutamate within the prelimbic and infralimbic cortices regulates the incubation of cocaine-seeking in rats. Neuropharmacology. 2018;128:293-300.

45. Cates HM, Li X, Purushothaman I, Kennedy PJ, Shen L, Shaham Y, et al. Genomewide transcriptional profiling of central amygdala and orbitofrontal cortex during incubation of methamphetamine craving. Neuropsychopharmacology. 2018 https://doi.org/10.1038/s41386-018-0158-x.

46. Li X, Rubio FJ, Zeric T, Bossert JM, Kambhampati S, Cates HM, et al. Incubation of methamphetamine craving is associated with selective increases in expression of Bdnf and trkb, glutamate receptors, and epigenetic enzymes in cue-activated fos-expressing dorsal striatal neurons. J Neurosci. 2015;35:8232-44.

47. Li X, Carreria MB, Witonsky KR, Zeric T, Lofaro OM, Bossert JM, et al. Role of dorsal striatum histone deacetylase 5 in incubation of methamphetamine craving Biol Psychiatry. 2018;84:213-22.

48. Li X, Witonsky KR, Lofaro OM, Surjono F, Zhang J, Bossert JM, et al. Role of anterior intralaminar nuclei of thalamus projections to dorsomedial striatum in incubation of methamphetamine craving. J Neurosci. 2018;38:2270-82.

49. Belin D, Everitt BJ. Cocaine seeking habits depend upon dopamine-dependent serial connectivity linking the ventral with the dorsal striatum. Neuron. 2008; 57:432-41. 